\title{
Professor Dr. Fridtjof Nüsslin zum 75. Geburtstag
}

\author{
Wolfgang Schlegel
}

Online publiziert: 27. August 2014

(C) Springer-Verlag Berlin Heidelberg 2014

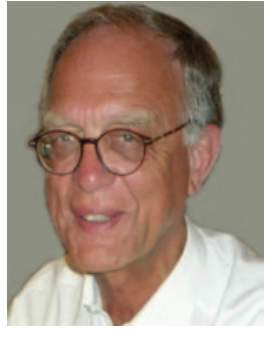

Fridtjof Nüsslin (Mit freundlicher Genehmigung, Wolfgang Schlegel)

Professor Fridtjof Nüsslin wurde am 30.07.1939 in Berlin geboren. Er studierte Physik in Tübingen und Heidelberg und promovierte mit einem kernphysikalischen Thema am Max-Planck-Institut für Kernphysik in Heidelberg. Im Jahr 1970 wechselte er in das Fachgebiet der Medizinischen Physik und war zunächst wissenschaftlicher Assistent in der Abteilung für Strahlentherapie der Medizinischen Hochschule Hannover, später leitender Medizinphysiker in der Klinik für Radioonkologie am Nord-West-Krankenhaus in Frankfurt/Main. Er habilitierte sich auf dem Gebiet der Medizinischen Physik in der Strahlentherapie und wurde1987 auf den Lehrstuhl für Medizinische Physik an der Universität Tübingen berufen. Dort leitete er bis 2004 die Abteilung für Medizinische Physik an der Uniklinik Tübingen. Nach seiner Emeritierung wechselte er als Gastprofessor für Biomedizinische Physik an die Klinik für

Prof. Dr. W. Schlegel $(\bowtie)$

Medizinische Physik in der Strahlentherapie,

Deutsches Krebsforschungszentrum,

Heidelberg, Deutschland

E-Mail: w.schlegel@dkfz.de
Strahlentherapie der TU München. Seit 2008 hat er eine „Distinguished Affiliate Professorship“ an der TU München inne. Außerdem ist er Mitglied am Institute for Advanced Studies der TU München.

Fridtjof Nüsslin hat die moderne Medizinische Physik in der Strahlentherapie entscheidend beeinflusst. Auf dem Weg zur Habilitation erarbeitete er viele, heute noch gültige Prinzipien der Dosimetrie und Bestrahlungsplanung und ermöglichte mit seinen Arbeiten die Einführung der Computertomographie in die Therapieplanung. Währendseiner Zeit in Tübingen hat er ein breites Spektrum medizin-physikalischer Probleme bearbeitet. Hervorzuheben sind wissenschaftliche Entwicklungen in der Konformationsstrahlentherapie, der stereotaktisch geführten Strahlentherapie und der IMRT. Monte-Carlo-basierte Dosisberechnungen, inverse Planung und bildgeführte adaptierte Strahlentherapie sind nur einige Beispiele, mit denen seine Abteilung höchste internationale Anerkennung erlangt hat. In München widmete er sich den Gebieten der biologischen und molekularen Bildgebung und den Modellen in der Tumorbiologie. Er war maßgeblich am Aufbau des Munich-Centers for Advanced Photonics (MAP) beteiligt, ein Exzellenzzentrum, das sich das Ziel gesetzt hat, Laserstrahlung in der biomedizinischen Bildgebung und zur Erzeugung von Teilchenstrahlung für die Strahlentherapie einzusetzen. Auch diese Forschungsarbeiten, denen er sich in München mit besonderer Begeisterung widmete, haben internationales Ansehen erlangt.

In besonderer Weise hat sich Fridtjof Nüsslin national und international für die wissenschaftliche und professionelle Festigung der Fachgebiete Medizinische Physik und Strahlentherapie eingesetzt. Als Präsident der Deutschen Gesellschaft für Medizinische Physik (DGMP), der European Federation of Organisations for Medical Physics (EFOMP) und der International Organisation for Medical 
Physics (IOMP) trieb er den weltweiten Aufbau der Medizinischen Physik voran und förderte nachhaltig die Aus- und Weiterbildung in diesem Bereich. Die Gründung der European School for Medical Physics (ESMP) in Archamps fällt in seine Amtszeit als EFOMP-Präsident, ebenso wie die Etablierung internationaler Medizin-Physik-Kongresse der EFOMP und der IOMP. Herausragendes hat Fridtjof Nüsslin ebenfalls als unermüdlicher Gutachter und Berater der DFG geleistet. Hier ist insbesondere die Gründung der Exzellenzakademie Medizintechnik (EAMT) der DFG zu nennen, die es dem wissenschaftlichen Nachwuchs ermöglicht, sich zu profilieren und Erfahrungen im Bereich der Wissenschaftsförderung und -organisation zu sammeln.

Seine Arbeit und seine Verdienste um die Medizinische Physik und Strahlentherapie wurden durch zahlreiche Ehrungen und Preise ausgezeichnet, darunter die Ehrenmitgliedschaft der DEGRO, der OEGRO und der EFOMP und die Glocker-Medaille der DGMP. Eine besondere Ehrung wurde ihm 2013 durch die IOMP zuteil, von der er als einer der 50 bedeutendsten Medizinphysiker der letzten 50 Jahre benannt wurde.

Fridtjof Nüsslin ist nicht nur ein verdienstvoller und anerkannter Wissenschaftler, sondern für viele von uns auch enger persönlicher Freund mit sprühendem Geist, Charme und Humor. Wir freuen uns auf weitere gemütliche abendliche Runden und spannende Gespräche mit ihm und seiner lieben Frau am Rande wissenschaftlicher Kongresse. Wir wünschen ihm den Erhalt seiner unermüdlichen Schaffenskraft, daneben aber auch Zeit für seine Hobbies und Feriendomizile, viel Freude an den Früchten seines Erfolges und gesundheitlich alles Gute.

Wolfgang Schlegel, Heidelberg 